\title{
Is ischemia modified albumin a good marker in acute exacerbation of chronic obstructive pulmonary disease?
}

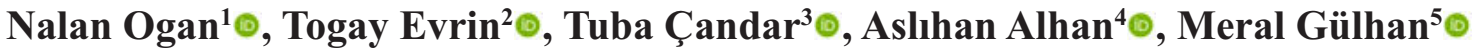 \\ ${ }^{1}$ Department of Chest Diseases, Ufuk University School of Medicine, Ankara, Turkey \\ ${ }^{2}$ Department of Emergency Medicine, Ufuk University School of Medicine, Ankara, Turkey \\ ${ }^{3}$ Department of Medical Biochemistry, Ufuk University School of Medicine, Ankara, Turkey \\ ${ }^{4}$ Department of Statistics, Ufuk University School of Medicine, Ankara, Turkey \\ ${ }^{5}$ Department of Chest Diseases, Hitit University School of Medicine, Çorum, Turkey
}

\section{ABSTRACT}

Objectives: Our aim is to compare the Ischemia modified albumin (IMA) in patients with acute exacerbation of Chronic Obstructive Pulmonary Disease (COPD) with a high oxidative and inflammatory biologic marker like $\mathrm{C}$-reactive protein (CRP) and to investigate its employability in AECOPD and the relationship between arterial blood gas and pulmonary function parameters.

Methods: Forty-six patients diagnosed with acute exacerbation of COPD between March 2015 - September 2016 at Ufuk University School of Medicine were included. The 1st and 5th days of IMA and CRP levels were measured. Also, IMA levels were given in absorbance units (ABSU).

Results: Total 46 patients of COPD, $13(28.3 \%)$ were females and $33(71.76 \%)$ were males. The mean age of the patients was $71.39 \pm 10.04$ years. The 1 st and 5 th day values of IMA, ABSU and CRP were $1.08 \pm 0.33$ and $0.49 \pm 0.24 ; 1.06 \pm 0.34$ and $0.49 \pm 0.26$; and $29.25(3.10-288.00)$ and $6.35(0.30-149.00)$, respectively $(p$ $<0.001)$. No significant correlation was determined between IMA and CRP. Also, no correlation were determined between the parameters of arterial blood gas and pulmonary function.

Conclusions: Although IMA values showed significant increase during acute exacerbation of COPD and decreased after treatment, CRP still appeared more effective in evaluating the exacerbation status and following up of the treatment of patients with COPD.

Keywords: Chronic obstructive pulmonary disease, exacerbation, C-reactive protein, ischemia modified albumin

$\mathrm{C}$ hronic obstructive pulmonary disease (COPD) is one of the leading causes of morbidity and mortality worldwide [1]. It is well-established that COPD is not only associated with pulmonary inflammation, but also with systemic inflammation. The mechanistic basis underlying COPD involve recurrent inflammation, oxidative stress, protease/antiprotease imbalance, environmental insult, and host genetics [2]. Increased plasma pro-inflammatory cytokines, hypoxia and increased oxidative stress may cause endothelium damage [3]. Acute exacerbation of COPD is an important event in the natural history of COPD that negatively impacts health status, increases the rates of hospitalization, and disease progression. COPD exacerbations are associated with increased airway and systemic inflammation and physiological changes, especially the

Received: January 25, 2019; Accepted: August 6, 2019; Published Online: January 30, 2020

How to cite this article: Ogan N, Evrin T, Çandar T, Alhan A, Gülhan M. Is ischemia modified albumin a good marker in acute exacerbation of chronic obstructive pulmonary disease? Eur Res J 2020;6(5):388-394. DOI: 10.18621/eurj.517778

Address for correspondence: Nalan Ogan, MD., Ufuk University School of Medicine, Department of Chest Diseases, Mevlana Bulvarl 86/88, 06520 Balgat, Ankara, Turkey.E-mail: nalanogan@gmail.com 
development of hyperinflation [4].

C-Reactive Protien (CRP) is an acute-phase protein produced by the liver in response to IL-6 stimulation. CRP is raised in most conditions associated with infection, inflammation, or tissue damage and it iswidely used for the assessment of acute exacerbation of COPD [5].

I schemia modified albumin (IMA) is a form of albumin modified by oxidative stress. The pathophysiological events of ischemia, including hypoxia and free oxygen radicals changed the N-terminus of albumin, and this molecule is called as IMA [6]. IMA was first discribed as a marker of myocardial ischemia, but now it has been shown that elevated in various states of noncardiac ischemia and oxidative stress conditions such as cerebrovascular diseases, inflammatory bowel diseases, chronic liver diseases, obstructive sleep apnea (OSA) and pulmonary embolism [7-13].Additionally, it was suggested to be a strong indicator of short-term mortality in patients with end-stage renal disease [14] and myocardial infarction with ST elevation [15].

Therefore the need for a useful biomarker to provide objective confirmation of exacerbation and predict the severity of COPD, we examinethe serum IMA, and compare between ABSU and CRP levels, in patients with acute exacerbation of COPD.

\section{METHODS}

The study was conducted on 46 acute exacerbation of COPD patients between March 2015- September 2016 applying to Emergency Unit of Ufuk University School of Medicine, Ankara, Turkey. Exclusion criteria were being under 18 years of age, the diagnosis of acute coroner syndrome, pulmonary emboli, acute or chronic renal failure and acute cerebrovascular disease and being reluctant to participate in the study, while inclusion criteria were being over 18 years old with diagnosis of COPD. Blood samples were taken from the patients on the first and the fifth day of IMA and CRP. CRP levels were measured by immunoturbidimetricassay (Abbott $^{\circledR}$ Architect c8000, USA). Hemogram parameters were measured with autoanalyzer (Abbott Cell-Dyn Ruby-Serial Number 54507BG/Abbott Diagnostics). Electrolyte measurements and the other biochemical parameter assays were performed with autoanalyzer (Abbott ${ }^{\circledR}$ Architect c8000, USA). The Architect cSystems ICT (Integrated Chip Techonology) is used for the quantitation of sodium, potassium and chloride in human serum. The Quantita D-Dimer assay were performed by the quantitative determination of D-Dimer in human citrated plasme using the Abbott ARCHITECT cSystems. The cholesterol and the other lipid parameter levels were performed for the quantitation of lipids in human serum with Abbott $^{\circledR}$ Architect c8000, USA. CK-MB and hsTnI levels were measured with autoanalyzer (Abbott ${ }^{\circledR}$ Architect i2000, USA). Architect STAT CKMB and STAT High Sensitive Troponin-I assays are a chemiluminescent microparticle immunoassay (CMIA) for the quantitative determination of the MB isoenzyme 3 of creatine kinase (CK-MB) and cardiac troponin I (cTnI) in human serum.

Body mass index (BMI) was calculated as weight divided by the square of the height $\left(\mathrm{kg} / \mathrm{m}^{2}\right)$. Modified British Medical Research Council (mMRC) Dyspnea Scale of patients were taken from patients' medical records at their latest polyclinic control during stable COPD period. The ethical commission approval was taken from the same hospital.

A $5 \mathrm{cc}$ of venous blood sampling was performed on all patients before treatment (for ischemia-modified albumin assay) and to non-anticoagulant biochemistry tubes with gel. After waiting for about 30 minutes for coagulation of the blood in the tubes, they were centrifuged at $4000 \mathrm{rpm}$ for 10 minutes. Serum samples were stored at $-80^{\circ} \mathrm{C}$ deep freeze. Ischemia modified albümin levels were analyzed by the difference in the capacity of albumin cobalt binding. A decrease in this abovementioned binding capacity was evaluated by a rapid and colorimetric assessment method developed by Bar-Or et al. [6]. The analyses in the spectrophotometer (Human HumalyzerVR 2000, Germany) were performed at $470 \mathrm{~nm}$ for the detection of absorbance of the specimens, and the results were given as absorbance units (ABSU). For the accurate estimation of maternal IMA levels, correction formula [individual serum albumin concentration/median albumin concentration of the population $\times$ IMA] defined by Lippi et al. [16]. Results were proportioned with serum albumin levels and expressed in terms of absorbance unit (ABSU)/g albumin. 
Pulmonary function tests (PFTs) results were taken from medical records of the patients at their latest polyclinic control. They were performed by "VMAX"; "Encore system (Germany)" device. During PFTs, post-bronchodilator FEV1\%, FVC\% and FEV1/FVC values were recorded based on GOLD criteria. For COPD staging, patients with FEV1 values $>80 \%$ were considered to have mild, FEV1: $80-50 \%$ were considered to have moderate, FEV1: $50-30 \%$ were considered to have severe, and FEV $1<30 \%$ were considered to have very severe COPD.

Arterial blood gase (ABG) analyses were performed by 2001 version of the "Instrumentation Laboratory-Synthesis 25 " device. $\mathrm{pH}$, partial oxygen pressure $(\mathrm{PaO} 2)$, partial carbon dioxide pressure $(\mathrm{PaCO} 2)$ and arterial oxygen saturation $(\mathrm{SaO} 2)$ values were recorded.

\section{Statistical Analysis}

All statistical analyses were performed by SPSS for Windows version 18.0 (SPSS Inc, Chicago, Illinois, USA). Definitive statistics were expressed as number $(\mathrm{N})$ and percentage $(\%)$ ratio. Proportions were compared with the chi-square test. Distribution of numeric values was evaluated by Kolmogorov Smirnov test. Data with non-normal distribution were expressed as median (minimum-maximum), while data with normal distribution were expressed as mean \pm standard Deviation. In the presence of comorbid diseases, comparisons of groups in pairs was made with the Student's t-test for data with normal distribution and Mann Whitney $U$ test for data with non-normal distribution. For comparisons of paired data, Paired-sample T-test was used for data with normal distribution, while Wilcoxon test was used for data with non-normal distribution. For correlation analyses, Pearson correlation coefficient or Spearman correlation coefficient was used in accordance with the status of data distribution. Reciever operator characteristics (ROC) curves were performed to evaluate the prognostic values of IMA and adj IMA for predicting acute exacerbation of COPD. A $p$ value $<0.05$ was considered statistically significant.

\section{RESULTS}

A total of 46 patients were included in the study, $13(28.3 \%)$ females and $33(71.76 \%)$ males. The mean age of the patients was $71.39 \pm 10.04$ years. The average package of cigarette smoked was $44.74 \pm$ 33.38 (Table 1). IMA, ABSU and CRP values were

Table 1. Demographic characteristics, number of annual attacks, mMRC score and BMI.

\begin{tabular}{lc}
\hline Characteristics & Data \\
\hline Sex (male/female), $\mathrm{n}(\%)$ & $33 / 13(71.7 / 28 / 3)$ \\
Mean age, years & $71.39 \pm 10.04$ \\
COPD group, n (\%) & $11(23.9)$ \\
C & $35(76.1)$ \\
D & \\
Smoking history (pack-year), n (\%) & $11(23.9)$ \\
never & $3(6.5)$ \\
current & $32(69.6)$ \\
Ex-smoker & $44.74 \pm 33.38$ \\
Smoking history (pack-year) & $11.59 \pm 6.55$ \\
Disease duration (years) & $1(0-5)$ \\
Number of annual attack, median (min-max) & $2.23 \pm 0.82$ \\
mMRC & $28.19 \pm 5.41$ \\
\hline BMI, kg/m ${ }^{2}$ &
\end{tabular}

Data are shown mean \pm standart deviation or $\mathrm{n}(\%)$. COPD $=$ chronic obstructive pulmonary disease, $\mathrm{mMRC}=$ Modified Medical Research Council, BMI = Body mass index 
Table 2. The values of CRP and IMA values on the first and the fifth days

\begin{tabular}{lccc}
\hline & $\mathbf{1}^{\text {st }} \mathbf{d a y}$ & $\mathbf{5}^{\text {th }}$ day & $\boldsymbol{p}$ value \\
\hline CRP (mg/dL) & $29.25(3.10-288.00)$ & $6.35(0.30-149.00)$ & $<\mathbf{0 . 0 0 1}$ \\
IMA (ABSU) & $1.08 \pm 0.33$ & $0.49 \pm 0.24$ & $<\mathbf{0 . 0 0 1}$ \\
IMA/albumin ratio (ABSU/g/dL) & $1.06 \pm 0.34$ & $0.49 \pm 0.26$ & $<\mathbf{0 . 0 0 1}$ \\
\hline
\end{tabular}

$\mathrm{CRP}=\mathrm{C}$ - reactive protein, $\mathrm{IMA}=$ Ischemia modified albumin, $\mathrm{ABSU}=$ Absorbance units

Table 3. The relationship between CRP value and IMA on the first day, disease progression, disease duration, number of annual attacks, arterial blood gas, biochemical tests, Echocardiography and pulmonary function tests.

\begin{tabular}{lcc}
\hline & $\mathbf{R}$ & $\boldsymbol{p}$ value \\
\hline IMA (ABSU) & 0.94 & 0.373 \\
\hline IMA/albumin ratio (ABSU/g/dL) & -0.078 & 0.606 \\
\hline Disease stage & -0.022 & 0.886 \\
\hline Disease duration (years) & 0.215 & 0.15 \\
\hline Annual attacks (n) & 0.30 & 0.84 \\
\hline BMI (kg/m ${ }^{2}$ & -0.238 & 0.111 \\
\hline Arterial pO2 (mmHg) & -0.310 & 0.036 \\
\hline Arterial pCO2 (mmHg) & 0.024 & 0.875 \\
\hline FEV1 (\%) & 0,034 & 0.823 \\
\hline FEV1/FVC $(\%)$ & -0.253 & 0.090 \\
\hline Troponin (ng/ml) & -0.079 & 0.604 \\
\hline CK (U/L) & -0.139 & 0.356 \\
\hline CK-MB (U/L) & -0.050 & 0.739 \\
\hline Albumin (g/dL) & -0.345 & 0.019 \\
\hline Total-C (mg/dL) & -0.077 & 0.964 \\
\hline LDL-C (mg/dL) & -0.048 & 0.741 \\
\hline HDL-C (mg/dL) & 0.018 \\
\hline Triglycerides (mg/dL) & -0.007 & 0.691 \\
\hline EF (\%) & -0.050 \\
\hline mPAP (mmHg) & -0.079 \\
\hline
\end{tabular}

$\mathrm{FEV} 1$ = forced expiratory volume in 1 second, FEV1/FVC $=$ Forced expiratory volume in 1 second /forced vital capacity, $\mathrm{CK}=$ creatin kinase, $\mathrm{CK}-\mathrm{MB}=$ creatin kinase-MB, HDL-C $=$ high-density lipoprotein cholesterol, LDL-C $=$ low-density lipoprotein cholesterol, Total- $\mathrm{C}=$ total cholesterol, $\mathrm{EF}=$ ejection fraction, $\mathrm{mPAP}=$ Mean pulmonary arteial pressure. 
shown on the first day and on the fifth day in Table 2. There was a significant decrease in all these values after treatment $(p<0.001)$. However, we could not find the same correlation for the admission day results of CRP and IMA among COPD stage, COPD years, and attack numbers of year (Table 3 ).

ROC analysis resulted a cut-off value for IMA level $\geq 0.845$ as higher inflammation with sensitivity $=0.79$ and specificity $=0.34$ (Fig. 1A). Additionally, ROC analysis resulted a cut-off value for adj IMA level $\geq 0.838$ as higher inflammation with sensitivity $=0.72$ and specificity $=0.29$ (Fig. 1B). ROC analysis showed that although the sensitivity of our test results was partially high their specificity to show exacerbation was low.

\section{DISCUSSION}

As our knowledge, our study is the first to investigate the IMA levels in patients with acute exacerbation of COPD before and after the treatment. The values of serum IMA, ABSU and CRP were assessed at first and fifth days and we observed that although there was no correlation between CRP and IMA and ABSU levels at the first day of hospital admission, a significant decrease was demonstrated for these values at the fifth day of treatment. The reason of this can be explained with IMA half-life being shorter than CRP and IMA is studied manually while CRP is studied with automatic standardized devices, leading to a possible discrepancy.

In COPD, especially in advanced stage and during exacerbations, various cytokines showing systemic inflammation, acute phase proteins, and chemokines are increased and abnormal changes in the circulating cells are observed [17]. Oxidative stress is defined as the decrease in anti-oxidant capacity against oxidants and/or the increase in oxidants. Oxidant matters form reactions with various biological molecules like protein, lipid, and nucleic acid and damage in the structure of extracellular matrix, biologic membranes, genetic cell structure by DNA damage, and ciliary function. Enzymatic events are affected, surfactant activity is decreased, and mucus production is increased along with the increase in the effectiveness of cytokines and proteases [18]. IMA is a modified serum albumin formed under conditions of oxidative stress and accepted as a biological marker of ischemia. Ischemic stress (like hypoxia, acidosis, and free radical damage), reactive oxygen radicals that appear during ischemia changes the N-terminal of the albumin and this results in the decrease of the binding ability of the albumin to nickel, cobalt and copper. It
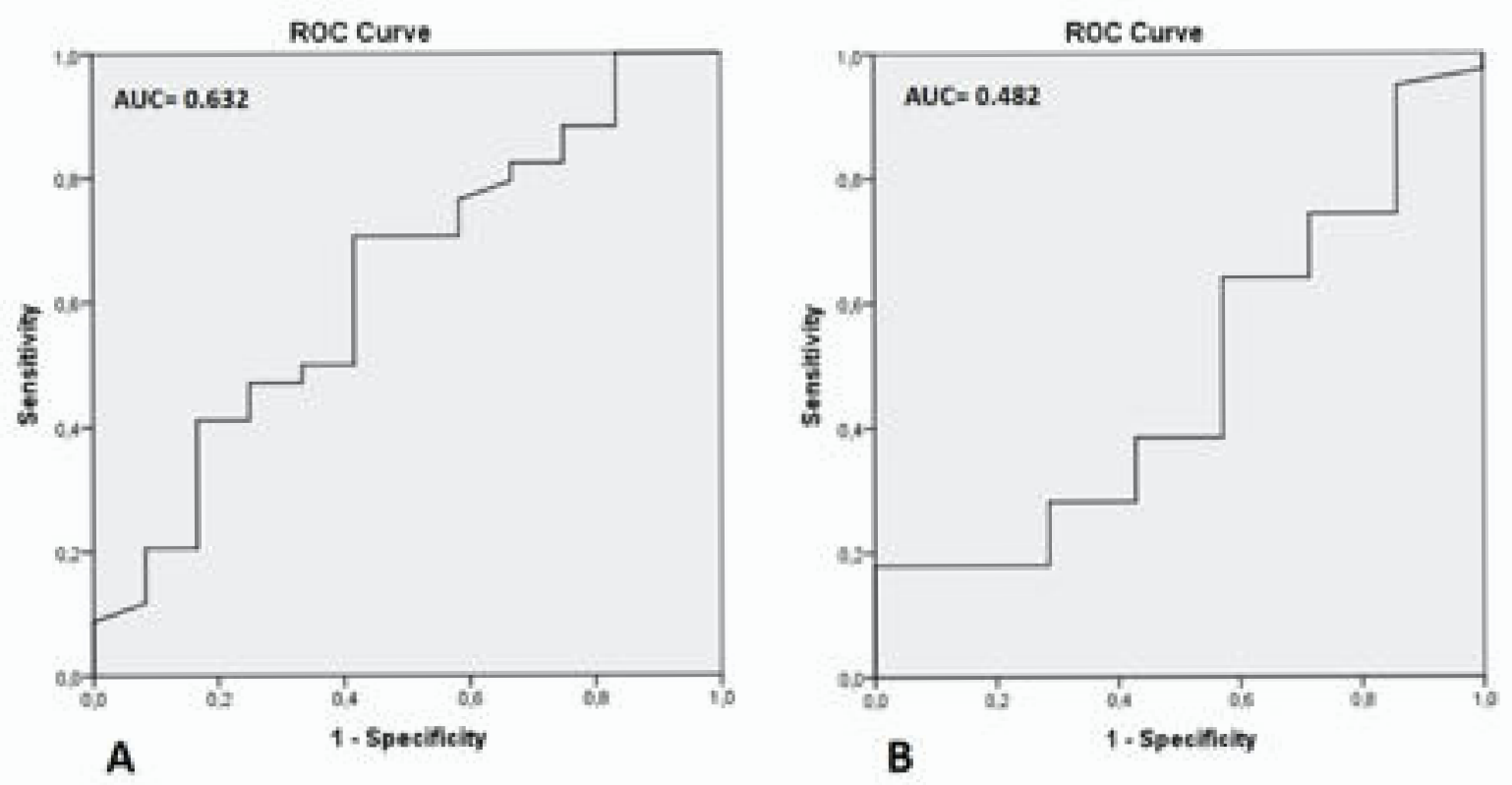

Fig. 1. ROC curve for IMA level (A), ROC curve for ABSU levels (B). 
increases in the few minutes after the start of ischemia, rises for 6-12 hours, and returns to normal in 24 hours [18]. Many studies have been published about IMA in acute cardiac and noncardiac ischemic events, however only few studies were related to COPD [713]. Can et al. [19] compared serum IMA, ox-LDL (Oxidized Low-Density Lipoprotein), TAS (Total Anti-oxidant Status), and TOS (Total Oxidant Status) levels in 51 patients with stable COPD and 45 healthy cases. Their results revealed that TAS levels did not show any difference between two groups while IMA, ox-LDL, and TOS levels were significantly increased in the COPD group, and furthermore ox-LDL and TOS levels were high only in stage IV while IMA levels were high in GOLD stages I, III, and IV. The author suggested that this finding indicated IMA being a marker showing hypoxia and oxidative stress in COPD [19]. Also, a study by Yang et al. [20], showed a significant correlation between the severity of COPD as per GOLD criteria and increased serum concentrations of oxidative parameters such as IMA. In the present study, IMA levels of COPD patients were compared on the day of admission because of exacerbation and on the $5^{\text {th }}$ day, which is relatively a more stable period, and it was found that IMA levels decreased along with the treatment response. This is indicative of decreased oxidative stress as the exacerbation is being controlled.

On the other hand, in the study of Roy et al. [21] with marathon runners, a decrease in IMA concentrations was observed immediately after exercise, and an increase after 24-48 h. Paulraj et al. [22] found low IMA values and positive correlation between the levels of IMA and the degree of smoking in their study. They explained decreased IMA levels are reason of a rise in lactate. Another reason could be low albumin levels that given falsely a low IMA value. Zapico-Muniz et al. [23] also revealed an immediate and transient decrease in IMA concentrations after the induction of forearm ischemia. In the present study, IMA and CRP levels at admission showed significant decrease as a response to treatment in patients with acute exacerbation of COPD. However, this correlation of CRP could not be detected with IMA and ABSU.

\section{Limitations}

One of the limitations of the present study is the limited number of patients. Second, no comparison with the stable period after discharge from hospital was made. Instead of this, control levels on the $5^{\text {th }}$ day were measured because of the short half-life of IMA. Furthermore, no comparisons with healthy controls were studied.

\section{CONCLUSION}

IMA is studied in many diseases, however, data regarding COPD is very limited. In accordance with the present findings, it can be stated that as the present patients had advanced age, mostly positive smoking history, and had a high rate of comorbidities, especially cardiac problems, IMA can be indicative of oxidative stress and possible complications in patients with COPD. The short half-life of IMA will accelerate the patients' scanning phase in terms of determining treatment priorities. Nonetheless, as IMA increases in many ischemic, neoplastic, and traumatic events, CRP still seems to be a cheaper and more efficient marker when compared with IMA. Further investigations are required for determining the employability of IMA in following-up the prognosis of COPD acute exacerbations.

\section{Conflict of interest}

The authors disclosed no conflict of interest during the preparation or publication of this manuscript.

\section{Financing}

The authors disclosed that they did not receive any grant during conduction or writing of this study.

\section{REFERENCES}

1. 2019 Global Strategy for Prevention, Diagnosis and Management of COPD. Available at: https://goldcopd.org/goldreports/. Accessed February 21, 2019.

2. Fischer BM, Pavlisko E, Voynow JA. Pathogenic triad in COPD: oxidative stress, protease-antiprotease imbalance, and inflammation. Int J Chron Obstruct Pulmon Dis 2011;6:413-21. 3. Rahman I, Morrison D, Donaldson K, MacNee W. Systemic oxidative stress in asthma, COPD, and smokers. Am J Respir Crit Care Med 1996;154:1055-60.

4. Wedzicha JA, Seemungal TA. COPD exacerbations: defining their cause and prevention. Lancet 2007;370:786-96.

5. Hurst JR, Donaldson GC, Perera WR, Wilkinson TM, Bilello 
JA, Hagan GW, et al. Use of plasma biomarkers at exacerbation of chronic obstructive pulmonary disease. Am J Respir Crit Care Med 2006; 174:867-74.

6. Bar-Or D, Lau E, Winkler JV. A novel assay for cobalt-albumin binding and its potential as a marker for myocardial ischemia-a preliminary report. J Emerg Med 2000;19:311-5.

7. Hazini A, Cemek M, Isıldak I, Alpdagtaş S, Onul A, Senel U, et al. Investigation of ischemia modified albumin, oxidant and antioxidant markers in acute myocardial infarction. Postep Kardiol Inter 2015;11:298-303.

8. Nepal M, Jaisawal S, Guragain M, Kafle P, Mukkera S, Kumar $\mathrm{R}$, et al. Ischemic modified albumin (IMA) as a novel marker for ischemic heart disease and surrogate marker for other high oxidative-ischemic conditions. J Cardiovasc Dis Res 2017;8:1126.

9. Jena I, Nayak SR, Behera S, Singh B, Ray S, Jena D, et al. Evaluation of ischemia-modified albumin, oxidative stress, and antioxidant status in acute ischemic stroke patients J Nat Sci Biol Med 2017;8:110-3

10. Guntas G, Sahin A, Duran S, Kahraman R, Duran I, Sonmez C, et al. Evaluation of Ischemia-Modified Albumin in Patients with Inflammatory Bowel Disease. Clin Lab 2017;63:341-7.

11. Kumar P, Subramanian K. The role of ischemia modified albumin as a biomarker in patients with chronic liver disease. $\mathrm{J}$ Clin Diagn Res 2016;10:9-12.

12. Ozben S, Huseyinoglu N, Hanikoglu F, Guvenc TS, Yildirim $\mathrm{BZ}$, et al. Advanced oxidation protein products and ischaemiamodified albumin in obstructive sleep apnea. Eur J Clin Invest 2014;44:1045-52.

13. Turedi S, Gunduz A, Mentese A, Topbas M, Karahan SC, Yeniocak S, et al. The value of ischemia-modified albumin compared with d-dimer in the diagnosis of pulmonary embolism. Respir Res 2008;9:1-12.

14. Sharma R, Gaze DC, Pellerin D, Mehta RL, Gregson H,
Streather CP, et al. Ischemia-modified albumin predicts mortality in ESRD. Am J Kidney Dis 2006;47:493-502.

15. Dominguez-Rodriguez A, Abreu-Gonzalez P, Jimenez-Sosa A, Samimi-Fard S, Idaira HB. Does ischemia-modified albumin add prognostic value to the thrombolysis in myocardial infarction risk score in patients with ST-segment elevation myocardial infarction treated with primary angioplasty? Biomarkers 2009; $14: 43-8$.

16. Lippi G, Montagnana M, Salvagno GL, Guidi GC. Standardization of ischaemia-modified albumin testing: adjustment for serum albümin. Clin Chem Lab Med 2007;45:261-2.

17. Barnes PJ, Celli BR. Systemic manifestations and comorbidities of COPD. Eur Respir J 2009;33:1165-85.

18. Barnes PJ, Shapiro SD, Pauwels RA. Chronic obstructive pulmonary disease: Molecular and cellular mechanisms. Eur Respir J 2003;22:672-88.

19. Can U, Yerlikaya FH, Yosunkaya S. Role of oxidative stress and serum lipid levels in stable chronic obstructive pulmonary disease. J Chin Med Assoc 2015;78:702-8.

20. Yang KY, Su VYF. Serum oxidative stress and chronic obstructive pulmonary disease. J Chin Med Assoc 2015;78:6878.

21. Roy D, Quiles J, Gaze DC, Collinson P, Kaski JC, Baxter GF. Role of reactive oxygen species on the formation of the novel diagnostic marker ischaemia modified albumin. Heart 2006;92:113-4.

22. Paulraj S, Kumar PA, Subramaniam K, Karthikeyan R. Role of oxidative stress in COPD. Can we use a novel biomarker to measure it? J Dent Med Sci 2017;16:52-6.

23. Zapico-Muniz E, Santalo-Bel M, Merce-Muntanola J, Montiel JA, Martinez-Rubio A, Ordonez-Llanos J. Ischemiamodified albumin during skeletal muscle ischemia. Clin Chem 2004;50:1063-5. 